\title{
Different Types of Epilepsy Based on Clinical and Electroencephalographic (EEG) Findings: Experience at Referral Neuroscience Hospital in Bangladesh
}

\author{
Mohammad Enayet Hussain ${ }^{1}$, AFM Al Masum Khan², Md Nahidul Islam², \\ Md. Ferdous Mian ${ }^{4}$, Md. Bakhtiar Azam5, Rajib Nayan Chowdhury ${ }^{6}$ \\ ${ }^{1}$ Associate Professor, Department of Neurology, National Institute of Neurosciences \& Hospital, Dhaka, Bangladesh; \\ ${ }^{2}$ Medical Officer, Department of Neurology, National Institute of Neurosciences \& Hospital, Dhaka, Bangladesh; \\ ${ }^{3}$ Assistant Professor, Department of Neurology, National Institute of Neurosciences \& Hospital, Dhaka, \\ Bangladesh; ${ }^{4}$ Assistant Professor, Department of Neurology, National Institute of Neurosciences \& \\ Hospital, Dhaka, Bangladesh; ${ }^{5}$ Assistant Professor, Department of Neurology, National Institute of \\ Neurosciences \& Hospital, Dhaka, Bangladesh; ${ }^{6}$ Associate Professor, Department of \\ Neurophysiology, National Institute of Neurosciences \& Hospital, Dhaka, Bangladesh
}

[Received: 21 January 2016; Revised: 6 March 2016; Accepted: 11 December 2016; Published: 1 January 2017]

\begin{abstract}
Background: A good history and a standard EEG recording help establish most of the epilepsy syndromes. Objective: The objective of this study was to establish different epilepsy syndromes on the basis of history and EEG in the clinically suspected seizure events. Methodology: This cross-sectional study was carried out in the neurophysiology laboratory of National Institute of Neurosciences \& Hospital, Dhaka, Bangladesh from January 2013 to December 2015, which included 2549 patients. EEG was obtained through surface scalp electrodes according to international 10/20 system. Patient and their attendants were interviewed using a semi structured questionnaire. The EEG findings, clinical history and in appropriate cases the neuroimaging, CSF and hematological findings were then correlated. Result: Among the 2549 patients most were children (39.8\% less than 10 years old) and young adult (30.63\% in 11 to 20 years age group). Male patients outnumbered female (63\% and $36 \%$ respectively). The overall sensitivity of EEG in yielding abnormal interictal epileptiform discharges was $42 \%$. About $32 \%$ of total 2549 patients were diagnosed as localization-related epilepsy (LRE), 5\% idiopathic generalized epilepsy (IGE), 1.41\% was Epileptic encephalopathy. Conclusion: In conclusion EEG is helpful in classifying the types of seizure, aids in defining the epilepsy syndrome, predicting the outcome and assists in management of patients. [Journal of National Institute of Neurosciences Bangladesh, 2017;3(1): 3-6]
\end{abstract}

Keywords: Interictal; EEG findings; type of epilepsy

Correspondence: Dr. Mohammad Enayet Hussain, Associate Professor, Department of Neurology, National Institute of Neurosciences \& Hospital, Sher-E-Bangla Nagar, Agargaon, Dhaka, Bangladesh; Email: enayetdmc@yahoo.com

Conflict of Interest: The authors declare that they have no competing interest.

Contributions to Authors: MEH was involved in EEG reporting, data analysis and writing the manuscript. RNC was involved in planning, consultation, EEG reporting and data collection for this study. The rest were involved in EEG reporting and data collection. All the authors have read and approved the final version of the manuscript.

Funding: This research project was not funded by any group or any institute on.

How to cite this article: Hussain ME, Khan AFMAM, Islam MN, Mian MF, Azam MB, Chowdhury RN. Different Types of Epilepsy Based on Clinical and Electroencephalographic (EEG) Findings: Experience at Referral Neuroscience Hospital in Bangladesh. J Natl Inst Neurosci Bangladesh, 2017;3(1): 3-6

Copyright: (C2017 Hussain et al. Published by Journal of National Institute of Neurosciences Bangladesh. This article is published under the Creative Commons CC BY-NC License (https://creativecommons.org/licenses/by-nc/4.0/). This license permits use, distribution and reproduction in any medium, provided the original work is properly cited, and is not used for commercial purposes.

\section{Introduction}

Seizure is any clinical event caused by abnormal electrical discharge in the brain, whilst epilepsy is tendency to have recurrent unprovoked seizure ${ }^{1}$. The pattern of epileptic discharges has been discovered by Gibbs and his colleagues ${ }^{2}$. Electroencephalogram (EEG) has been used to diagnose, classify and manage epilepsy for long time. There is wide variation of incidence of epilepsy worldwide due to variation in classification system of epilepsy and methodology adopted in different studies ${ }^{3}$.

The life time incidence of epilepsy varies from $2 \%$ to $5 \%$ 
(WHO report on Epilepsy in South East Asia)4. With the incidence of 2 to 10 per thousand for South East Asian countries, it is estimated that there are 1.5-2 million people suffering from epilepsy in Bangladesh ${ }^{4}$. The incidence is highest at both extreme of ages, especially in neonatal period and after 6th decade 5 . EEG still remains a very important investigation despite a tremendous advancement in neurodiagnostic procedures. The aim of this study was to determine the types of epilepsy on the basis of history and interictal EEG changes of patients referred to neurophysiology lab.

\section{Methodology}

This cross-sectional study was carried out in Neurophysiology laboratory of National Institute of Neurosciences \& Hospital, Dhaka, Bangladesh from January 2013 to December 2015 for a period of two (02) years. Meticulous history from the patient and the witness of the event was taken and proper physical examination was done by neurologists. All the information were kept in record files. We selected all the cases which were sent for EEG. Most patients were sleep deprived for at least 6 hours overnight except the new born and infants. EEG recordings were obtained through digital machine with minimal duration of 20 30 minute with electrode placed on scalp according to international $10 \sim 20$ systems. Recording was done preferably in both awake and sleeping state. Only awake recording was done in patient who did not sleep during the recording. In the mentally retarded and noncooperative patients only sleep recording was done. Provocative stimuli like photic stimulation were given to all patients. Hyperventilation was done in most cases if not all. The background activity was classified as normal (organized and symmetrical) or abnormal (disorganized and/or asymmetrical). The EEG was interpreted by consultant neurologist, trained in reading EEG. The EEG was examined for abnormal slow waves and specific epileptiform abnormality, the interictal spike or sharp wave. The abnormal electroencephalographic activity was also classified as generalized or focal. The presence and topography of abnormal slow waves and epileptiform discharges were evaluated. The latter were classified as spike/spike-and-wave, sharp/sharp-and-slow wave and polyspike/polyspikes-and-wave. Though a total of 2573 patients were initially interviewed, 24 patients with marked artifacts on EEG were excluded from this study.

\section{Results}

The study population included 2549 epilepsy patients. In this study, the patient's age ranged from 1 day to 81years. The mean age at presentation for doing EEG was $9 \pm 2.36$ years i.e. most of the patients were less than 10 years old $(39.78 \%)$. The next common age group at seizure presentation was 11 to 20 years $(30.63 \%)$. Only 11 patients $(0.70 \%)$ were older than 70 years (Table 1).

Table 1: Distribution of patients according to age

\begin{tabular}{lcc}
\hline Age Group & Frequency & Percentage \\
\hline 0 to 10 Years & 1014 & 39.78 \\
11 to 20 Years & 781 & 30.63 \\
21 to 30 Years & 404 & 15.84 \\
31 to 40 Years & 163 & 6.30 \\
41 to 50 Years & 90 & 3.53 \\
51 to 60 Years & 68 & 2.66 \\
61 to 70 Years & 18 & 0.70 \\
More Than 70 Years & 11 & 0.70 \\
Total & $\mathbf{2 5 4 9}$ & $\mathbf{1 0 0 . 0}$ \\
\hline
\end{tabular}

Table 2: Distribution of patients according to sex

\begin{tabular}{lcc}
\hline Gender & Frequency & Percentage \\
\hline Male & 1613 & 63.28 \\
Female & 936 & 36.72 \\
Total & $\mathbf{2 5 4 9}$ & $\mathbf{1 0 0 . 0}$ \\
\hline
\end{tabular}

Sixty three $(63 \%)$ percent of the patients were male and $37 \%$ were female (Table 2 ).

Table 3: Distribution of patient according to EEG findings

\begin{tabular}{lcc}
\hline Pattern of EEG & Frequency & Percentage \\
\hline Normal EEG & 1489 & 58.41 \\
Abnormal EEG & 1060 & 41.59 \\
Total & $\mathbf{2 5 4 9}$ & $\mathbf{1 0 0 . 0}$ \\
\hline
\end{tabular}

Out of 2549 EEGs done in clinically suspected cases, $1489(58.4 \%)$ were normal and $1060(41.6 \%)$ revealed abnormality (Table 3).

Table 4: Distribution as per the pattern of EEG abnormality

\begin{tabular}{lcc}
\hline Pattern & Frequency & Percentage \\
\hline GE & 131 & 5.13 \\
LRE & 827 & 32.44 \\
Epileptic Encephalopathy & 36 & 1.41 \\
Other Types & & \\
- NCSE & 04 & 0.15 \\
- SSPE & 39 & 1.53 \\
- FCD & 15 & 0.58 \\
- DCD & 08 & 0.31 \\
\hline
\end{tabular}

*NCSE $=$ Non-convulsive status epilepticus; $\mathrm{SSPE}=$ Subacute sclerosing panencephalitis; $\mathrm{DCD}=$ Diffuse Cerebral Dysfunction; $\mathrm{FCD}=$ Focal Cerebral Dysfunction 
EEG positivity in detecting electroencephalographic alteration in clinically suspected epileptic patients was 41.6\%. Of the 1060 abnormal EEG, 827 (78.01\%) was diagnosed as focal epilepsy, 131(12.35\%) had generalized (GE), epileptic encephalopathy in 36 $(3.39 \%)$ and rest $66(6.22 \%)$ had other diagnoses (Table 4).

Table 5: Distribution of epileptiform discharges according to site

\begin{tabular}{lcc}
\hline Type & Frequency & Percentage \\
\hline Temporal & 300 & 11.76 \\
Frontal & 262 & 10.27 \\
Parietal & 18 & 0.70 \\
Occipital & 23 & 0.90 \\
Central & 55 & 2.15 \\
Multifocal & 35 & 1.37 \\
Mixed & 116 & 4.55 \\
Benign & & \\
• BRE & 11 & 0.43 \\
• BOE & 07 & 0.27 \\
Total & $\mathbf{8 2 7}$ & $\mathbf{3 2 . 4 4}$ \\
\hline
\end{tabular}

Among the 827 patients with focal epilepsy, most common focus was temporal lobe $(300,36.27 \%)$, followed by frontal lobe $(262,10.27 \%)$ (Table 5). [BRE- Benign Rolandic Epilepsy, BOE- Benign Occipital Epilepsy]

Table 6: Generalized discharges

\begin{tabular}{lcc}
\hline Type of Generalized Epilepsy & Frequency & Percentage \\
\hline IGE & 94 & 3.68 \\
Absence & 25 & 0.98 \\
- Childhood & 18 & \\
- Juvenile & 06 & \\
- With Eyelid Myoclonia & 01 & \\
JME & 12 & 0.47 \\
Total & $\mathbf{1 3 1}$ & $\mathbf{5 . 1 3}$ \\
\hline
\end{tabular}

Among the 131 generalized epilepsy, IGE was commonest (94: 71.7\%), Absence \& JME was (25: $19.08 \%),(12: 9.16 \%)$ respectably (Table 6). [IGEIdiopathic Generalized Epilepsy, JME- Juvenile Myoclonic Epilepsy]

Of the 36 epileptic encephalopathy, $05(0.19 \%)$ had early infantile epileptic encephalopathy (EIEE) with suppression-burst, $08 \quad(0.31 \%)$ severe infantile myoclonic epilepsy, $08(0.31 \%)$ West syndrome, 05 (0.19\%) Lennox-Gastaut syndrome (LGS), 02 (0.07\%) Landau-Kleffner syndrome (LKS) and $08(0.31 \%)$ patients were found to have continuous spike wave of slow sleep (CSWS) (Table 7).

Table 7: Epileptic encephalopathy subtypes

\begin{tabular}{lcc}
\hline Type & Frequency & Percentage \\
\hline Early Infantile epileptic & 05 & 0.19 \\
encephalopathy with & & \\
SB/Otahara syndrome & & \\
Severe infantile myoclonic epilepsy & 08 & 0.31 \\
West syndrome & 08 & 0.31 \\
Lannox - gastaut syndrome & 05 & 0.19 \\
LK syndrome & 02 & 0.07 \\
CSWS & 08 & 0.31 \\
Total & $\mathbf{3 6}$ & $\mathbf{3 . 3 9}$ \\
\hline
\end{tabular}

In addition we found $4(0.15 \%)$ non-convulsive status epilepticus (NCSE), 39 (1.53\%) subacute sclerosing panencephalits (SSPE), $15(0.58 \%)$ focal cerebral dysfunction (FCD) and $8(0.31 \%)$ diffuse cerebral dysfunction (Table 4).

\section{Discussion}

Epileptiform activity is quite specific for a diagnosis of epilepsy as the cause of transient loss of consciousness or other paroxysmal event which is clinically likely to be epilepsy. In epileptic patients the EEG is examined for a specific epileptiform activity- the interictal spike/polyspikes or sharp wave. These discharges may either be focal or generalized. As most epileptic syndromes are common in the pediatric age group, most of the patients in our study were children. Like our study, most of the reports regarding sensitivity of EEG are retrospective evaluation of data base from tertiary care centers ${ }^{6-8}$.

Several published studies on adult epilepsies showed that the chance of detecting interictal epileptiform discharges (IEDs) from the first EEG varies between $29.0 \%$ and $55.0 \%$ at outdoor monitoring of patients ${ }^{6-8}$. Repeated EEG ultimately demonstrated the IEDs in $80.0 \%$ to $90.0 \%$ of the patients ${ }^{6,8}$. Video EEG monitoring also yielded similar results. Long term video EEG monitoring can detect IEDs in up to $81 \%$ of the patients. Adoption of several methods can increase the chance of detecting IEDs. Sleep effectively improves detection of both generalized and focal IEDs $^{10-12}$. The yield of epileptiform discharges increases if recording is done early after a seizure event ${ }^{11}$. Hyperventilation and photic stimulation also induces IEDs in many patients, especially in generalized seizures $^{13-15}$. In a series of 2648 patients with unquestionable diagnosis of seizure by Kershman et $\mathrm{al}^{16}, 46.5 \%$ had focal changes while $15.0 \%$ had diffuse 
generalized abnormality in their scalp recordings of EEG. In the present study the focal and generalized discharges were $32.44 \%$ and $5.13 \%$ respectively.

There are some limitations. Firstly, there is chance of sampling bias. Moreover, the chance of inter observer biasness was minimized by following same principle in recording and typing the EEG abnormality.

\section{Conclusion}

The present study showed that overall sensitivity of EEG in yielding abnormal interictal epileptiform discharges is good if the standard recording protocol is followed. Most of the abnormal EEG revealed focal discharges. The presence of focal IEDs suggests the diagnosis of a localization related epilepsy, the character and location of which offer clues to both the etiology of the epilepsy syndrome and the location of the epileptogenic region. Finding of a generalized IED suggests the diagnosis of one of the generalized epilepsy syndromes. Thus the interictal EEG serves several purposes, especially aids in differentiating true and pseudoseizures, helps in classifying the type of seizure disorder and also defining the epilepsy syndromes. Following the standard recording protocol through scalp electrodes it can detect abnormal cerebral neuronal discharges in two third cases of clinical seizure events. The finding of this study further consolidated the concept that EEG still remains the key investigation in clinically suspected epileptic patients and has a high level of sensitivity when performed in appropriate cases.

\section{References}

1. Allen CMC, Lueck CJ, Dennis M. Neurologic disease. In: Colledge NR, Walker BR, Ralston SH (eds) Davidson's Principles and Practice of Medicine, 21st edn. Elsevier Limited, 2010;1172 2. Gibbs FA, Davis H, Lennox WB. The electroencephalogram in epilepsy and in conditions of impaired consciousness. Arch Neurol Psychiatry 1935;34:1133-48

3. Yacubian EMT. Epilepsia: o conceito atual. In: Yacubian EMT (ed) Epilepsia da Antiguidade ao Segundo Milênio. Lemos, São Paulo, 2000;pp 82-8

4. WHO report on Epilepsy in South East Asia, Some facts and figures in Epilepsy; 1999; [Available at: http://www.searo.who.int/LinkFiles/Information and Documents facts]

5. Marino R Jr, Cukiert A, Pinho E. Aspectos epidemiológicos da epilepsia em São Paulo: um estudo de prevalência. Arq Neuro Psiquiatr 1986;44(3):243-54

6. Marsan CA, Zivin LS. Factors related to the occurrence of typical paroxysmal abnormalities in the EEG records of epileptic patients. Epilepsia 1970;11:361 -81

7. Goodin DS, Aminoff MJ, Laxer KD. Detection of epileptiform activity by different noninvasive EEG methods in complex partial epilepsy. Ann Neurol 1990;27:330-4

8. Salinsky M, Kanter R, Dasheiff RM. Effectiveness of multiple EEGs in supporting the diagnosis of epilepsy: an operational curve. Epilepsia 1987;28:331 -4

9. Walczak TS, Scheuer ML, Resor S et al. Prevalence and features of epilepsy without interictal epileptiform discharges. Neurology 1993;43(suppl):287-8

10. Angeleri F. Partial epilepsies and nocturnal sleep. In: Levin P, Koella W (eds) Sleep. Karger, Basel, 1975;pp 196-203

11. Niedermeyer E, Rocca U. The diagnostic significance of sleep electroencephalograms in temporal lobe epilepsy. A comparison of scalp and depth tracings. Eur Neurol 1972;7:119-29

12. Sato S, Dreifuss FE, Penry JK. The effect of sleep on spike-wave discharges in absence seizures. Neurology 1973;23:1335-45

13. Sato S, Dreifuss FE, Penry JK et al. Long-term follow-up of absence seizures. Neurology 1983;33:1590-5

14. Miley CE, Forster FM. Activation of partial complex seizures by hyperventilation. Arch Neurol 1977;34:371 -3

15. Wolf P, Goosses R. Relation of photosensitivity to epileptic syndromes. J Neurol Neurosurg Psychiatry 1986;49:1386-9

16. Kershman J, Vasques J, Golstein S. The incidence of focal and non-focal EEG abnormalities in clinical epilepsy. Electro encephal Clin Physiol 1951;3:15-24. 